\title{
Quantum Anomalies (in) Matter
}

\author{
Karl Landsteiner ${ }^{1, *}$ \\ ${ }^{1}$ Instituto de Física Teórica UAM-CSIC \\ C/ Nicolás Cabrera 13-15 \\ 28049 Campus Cantoblanco \\ SPAIN
}

\begin{abstract}
Over the last decade it has bee realized that triangle anomalies give rise to dissipationless transport phenomena in hot and dense relativistic matter. I will review anomalous transport theory and then discuss its applications to the quark gluon plasma and the electronics of Weyl semimetals. Finally I briefly discuss the absence of genuine chiral torsional transport.
\end{abstract}

\section{Introduction}

Anomalies are one of the most emblematic properties of quantum field theories. An anomaly appears when not all the symmetries of the classical theory can be realized in the quantum theory $[1,2]$. In four dimensional quantum field theory anomalies can be calculated in perturbation theory from Feynman diagrams of triangular shape. There one often speaks of triangle anomalies. For a general theory containing chiral fermions The general form of triangle diagrams can be summarized in the formulas

$$
\begin{aligned}
\left\langle D_{\mu} J_{a}^{\mu}\right\rangle & =\epsilon^{\mu \nu \rho \lambda}\left(\frac{d_{a b c}}{32 \pi^{2}} F_{\mu \nu}^{b} F_{\rho \lambda}^{c}+\frac{b_{a}}{768 \pi^{2}} R_{\beta \mu \nu}^{\alpha} R_{\alpha \rho \lambda}^{\beta}\right), \\
\left\langle D_{\mu} T^{\mu \alpha}\right\rangle & =F_{a}^{\alpha \mu} J_{\mu}^{a}+\epsilon^{\mu \nu \rho \lambda} \frac{b_{a}}{384 \pi^{2}} D_{\beta}\left(F_{\mu \nu}^{a} R_{\rho \lambda}^{\alpha \beta}\right) .
\end{aligned}
$$

Here we assume that the chiral fermions transform under a symmetry group generated by $T_{a}$. The theory is coupled covariantly to the sources for the currents $J_{a}^{\mu}$ and the energymomentum tensor $T^{\mu \nu}$. These classical sources are the gauge fields $A_{\mu}^{a}$ and the metric $g_{\mu \nu}$. An anomaly is present if it is not possible to define currents and energy-momentum tensor such that the operators obey the Ward identities corresponding to the classical symmetries. Anomalies are properties of the theory itself. Their presence or absence does not depend on applying external electromagnetic gauge fields or putting the theory in a non-trivial geometry. Indeed the presence of the anomaly can be calculated by a triangle diagram in flat space in the absence of external gauge fields. It is uniquely determined by the non-vanishing of the anomaly coefficients

$$
\begin{aligned}
d_{a b c} & =\operatorname{str}\left(T_{a} T_{b} T_{c}\right)_{\text {right }}-\operatorname{str}\left(T_{a} T_{b} T_{c}\right)_{\text {left }}, \\
b_{a} & =\operatorname{tr}\left(T_{a}\right)_{\text {right }}-\operatorname{tr}\left(T_{a}\right)_{\text {left }},
\end{aligned}
$$

*e-mail: karl.landsteiner@csic.es 
where str denotes the symmetrized trace and the subscripts denotes right- and left-handed fermion representations. In the case where only $U(1)$ symmetries are present the traces becomes just the sums over the (products) of charges, $d_{a b c}=\sum_{\text {right }} q_{a} q_{b} q_{c}-\sum_{\text {left }} q_{a} q_{b} q_{c}$ and $b_{a}=\sum_{\text {right }} q_{a}-\sum_{\text {left }} q_{a}$. The anomaly equations (1),(2) are operator equations thus independent of the state. They hold at finite temperature and chemical potential in the same form.

\section{Anomaly induced currents}

Since anomalies are understood as properties of the operators one could have naively expected that there is no additional effect due to anomalies depending on the state of the quantum system. This however is not true. It is very well established by now that anomalies give rise to new contributions to the currents and the energy-momentum tensor in a formal equilibrium state described by non-vanishing chemical potentials and at finite temperature (see [3] for a review and references therein for a guide to the literature)

$$
\begin{aligned}
& \left\langle\vec{J}_{a}\right\rangle=\frac{d_{a b c}}{4 \pi^{2}} \mu_{b} \vec{B}_{c}+\left(\frac{d_{a b c}}{4 \pi^{2}} \mu_{b} \mu_{c}+\frac{b_{a}}{12} T^{2}\right) \vec{\omega} \\
& \left\langle\vec{J}_{\epsilon}\right\rangle=\left(\frac{d_{a b c}}{8 \pi^{2}} \mu_{b} \mu_{c}+\frac{b_{a}}{24} T^{2}\right) \vec{B}_{a}+\left(\frac{d_{a b c}}{6 \pi^{2}} \mu_{a} \mu_{b} \mu_{c}+\frac{b_{a}}{6} \mu_{a} T^{2}\right) \vec{\omega} .
\end{aligned}
$$

The indexes number the symmetries, $\mu_{a}$ are chemical potentials corresponding to the symmetries numbered by $a^{1}$. The magnetic fields $\vec{B}_{a}$ are the given by the curl of the source $\vec{\nabla} \times \overrightarrow{A_{a}}$ for the current $\vec{J}_{a}$ and $\omega=\vec{\nabla} \times \vec{v}$ is the curl of the velocity. We note that the latter can be understood as the chemical potentials for the conserved momenta. The state of the system is thus describe the the state variables $T, \mu_{a}, \vec{v}$. Non of these appear in the fundamental Hamiltoniandescribing the quantum dynamics of the theory. In contrast the magnetic fields $\vec{B}_{a}$ are external couplings that do enter the Hamiltonian. Sometimes it is possible to substitute (gradients) of state variables by external fields. For example it is well known that a (the negative) gradient of a chemical potential has the same effect as an electric fields or the gradient of temperature has the same effect as a gradient in gravitational potential [4]. In the same vain the vorticity in eqs. (5), (6) can be substituted by a gravito-magnetic field [5]. This allows to calculate the anomalous currents in linear response theory via Kubo formulas and to pin down the appearance of the anomaly coefficients. Its a strong hint that the anomalies Eqs. (1) and (2) are the underlying deeper reason for these currents. Indeed this is by now well established. The dependence on the chemical potentials is fixed by the anomaly through by demanding a generalized second law to hold $[6,7]$ in the presence of anomalies. The temperature dependence can be fixed by geometrical arguments $[8,9]$. We note that in the latter case it involves integration of the anomaly equations in a black hole geometry and ties the anomaly induced currents tightly to the phenomenon of Hawking radiation [10]. An argument based on global anomalies has also been presented in [11].

These formulas describe the spontaneous generation of currents at finite temperature $T$ and chemical. These are only well defined in thermal equilibrium. We speak of a global symmetry if the current $J_{a}^{\mu}$ is not coupled to dynamical gauge fields whose dynamics is governed by gauge invariant Maxwell or Yang-Mills actions. For such a global current a spatially homogeneous expectation value is actually no an observable quantity. Such a current is only observable due to its divergence $\vec{\nabla} \cdot \vec{J}_{a}$ which describes accumulation of depletion of the global charge in a local manner.

\footnotetext{
${ }^{1}$ For non-Abelian symmetries they necessarily lie in the Cartan subalgabra.
} 
If the current is a true gauge current (as happens for the electric current) then a theorem originally due to Bloch ${ }^{2}$ states that the expectation value has to vanish exactly. At first sight this is puzzling since the anomaly coefficient $d_{A V V}$ which describes the axial anomaly $\partial J_{A}^{\mu}=\frac{1}{16 \pi^{2}} \epsilon^{\mu \nu \rho \lambda} F_{\mu \nu} F_{\rho \lambda}$ suggest the chiral magnetic effect $\vec{J}=\frac{\mu_{5}}{2 \pi^{2}} \vec{B}$, with $\mu_{5}$ being the chemical potential for the axial charge. Even such a homogeneous current would be observable because it would generate a magnetic field according to amperese law. By now it is however well known that the proper strict equilibrium expression contains a contribution arising from a counterterm needed to insure gauge invariance. Including this counterterm the chiral magnetic effect is described by

$$
\vec{J}=\frac{\mu_{5}-A_{0}^{5}}{2 \pi^{2}} \vec{B},
$$

where $A_{5}^{\mu}$ is the temporal component of the axial gauge field acting as source for the axial current. Defining strict equilibrium by the condition $A_{0}^{5}=\mu_{5}$ we see that indeed the chiral magnetic effect vanishes with this strict and narrow definition of equilibrium [13].

This makes it clear that the anomaly induced currents can only lead to observable effects if a certain amount of non-equilibrium physics is present which nevertheless can still be described by (possibly space and time dependent) chemical potentials and temperature. Such situations are commonly referred to a as local thermal equilibrium. We expect then the effects of the anomaly induced currents are important in situations that can by described in hydrodynamic theory, i.e. an effective theory of dynamics of (approximately) conserved charged densities at in the limit in which gradients of these densities are small compared to the mean free path.

One aspect of the anomaly induced currents is especially worth emphasizing. Contrary to the actual anomalies eqs. (1) and (2) the expression for these currents do not depend on the presence of external electric and magnetic fields or of spacetime curvature! This means that if one can drive a system of quantum matter containing chiral fermions into a state of local thermal equilibrium in which the currents eqs. (5) and (6) apply one can probe for anomalies (even gravitational ones) by means of transport measurments! They truly represent quantum anomalies in matter.

Now we briefly will discuss two examples of such quantum matter systems: heavy ion collisions and Weyl semi-metals

\section{Heavy Ion Collisions}

In heavy ion collisions as performed at the Relativistic Heavy ion Collider RHIC and the Large Hadro Collider LHC ions are collided at high energies in order to create a new state of matter: the quark gluon plasma. In the initial fire ball of the collision very high energy densities are achieve and the subsequent time evolution of the quark gluon plasma can be described by relativistic hydrodynamics. In non-central collisions a strong magnetic field is generated by the spectators not participating in the collision. The third ingredient is the vacuum structure of QCD and the gluonic part of the axial anomaly

$$
\partial_{\mu} J_{5}^{\mu}=\frac{N_{f}}{16 \pi^{2}} \epsilon^{\mu \nu \rho \lambda} \operatorname{tr}\left(G_{\mu \nu} G_{\rho \lambda}\right) .
$$

Topologically non-trivial gluon field configurations can be excited at high energies (so called strong sphalerons) and by the axial anomaly they will induce a non-vanishing axial charge. In such a chirally imbalanced medium the chiral magnetic effect is expected to lead to a signal

\footnotetext{
${ }^{2}$ See [12] for a modern formulation based on gauge symmetry.
} 
of charge separation along the direction of the magnetic field $[14,15]$. We note that this is a true non-equilibrium realization of the chiral magnetic effect. The chiral charge is generated by the strong sphalerons at an early stage [16]. However it also raises the question if the chiral magnetic current is immediately effective even far from equilibrium.

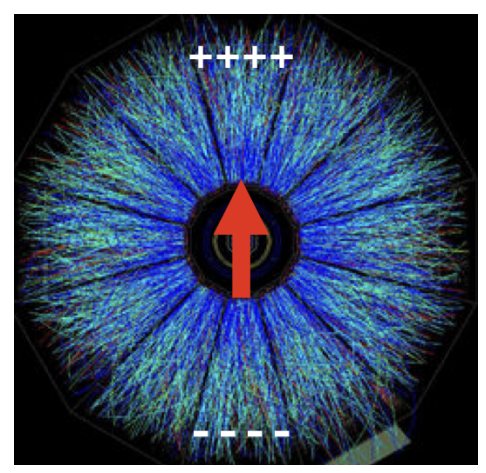

Figure 1. Particles are created in an heavy ion collision. The strong magnetic field at the early stages can lead to charge separation along the magnetic field (red arrow) via the Chiral Magnetic Effect.

The gauge/gravity duality (holography) has played a major role in the establishment and deeper understanding of anomaly induced transport phenomena. Furthermore it has become a standard tool to model hydrodynamics and far from equilibrium evolution of strongly coupled quantum systems. Therefore it is natural to investigate the question how fast the chiral magnetic current builds up in a holographic setup. This has been recently investigated in [17].

The results from this study indicate that the chiral magnetic current indeed shows a delayed build up. Adjusting the parameters of the holographic model to reproduce the entropy and the anomaly of three flavor QCD the build up times for typical RHIC energies can be estimated. For typical RHIC energies densities it is $\tau \approx 0.38 \mathrm{fm} / \mathrm{c}$ whereas for typical LHC energies it turns out to be $\tau \approx 0.114 \mathrm{fm} / \mathrm{c}$. This is high significance since the estimated lifetimes of the magnetic field at RHIC is $\tau_{B} \approx 0.6 \mathrm{fm} / \mathrm{c}$ and at LHC energies $\tau_{B}=0.02 \mathrm{fm} / \mathrm{c}$. This is a strong hint that the CME will not be observable at the higher LHC energies simply because the magnetic field decays before the chiral magnetic current exists. On the other hand at the lower RHIC energies there seems to be no obstacle for the CME to be effective.

In view of the recent discussion about the ambigious results of the RHIC isobar run [18] it is clear that a much better understanding of the chiral magnetic effect in the dynamical setup of a heavy ion collision is needed. Holographic toy models of the strongly coupled quark gluon plasma can be a very useful tool in this respect.

\section{Weyl Semimetals}

Weyl semimetals are a new class of materials whose low energy electronic quasiparticle excitations can be described by a Dirac equations [19]. In Figure 2 we show a schematic view of the band structure of a Weyl semi-metal. In this simple description, the band structure has two band crossing points near the Fermi level. By the Nielsen-Ninomiya theorem [20] the two nodal points have opposite chirality. To lowest order in a derivative expansion near the Fermi points, the electronics can be effectively described by two Weyl fermions of opposite chirality. A useful equivalent view is to combine both Weyl fermions into one Dirac fermion. 
Then the Dirac like wave equation for this four-component spinor is

$$
\left(i \gamma^{\mu} \partial_{\mu}+\gamma^{\mu} \lambda_{\mu} \gamma_{5}\right) \psi=0
$$

The four vector $\lambda_{\mu}=\left(\lambda_{0}, \vec{\lambda}\right)$ describes the separation of the Weyl nodes of opposite chirality in momentum space. A useful view is to consider $\lambda_{\mu}$ to be the background value of an axial gauge field $A_{\mu}^{5}=\lambda_{\mu}$. Now we can immediately see that in equilibrium eq. (7) applies and with $\mu_{5}=A_{0}^{5}=\lambda_{0}$ results in a vanishing chiral magnetic effect in equilibrium. Here it is important to note that the relativistic convention for defining the chemical potential applies. This means that the chemical potentials have to be measured with respect to the band-crossing points and not with respect to the bottom of band in the Brillouin zone.

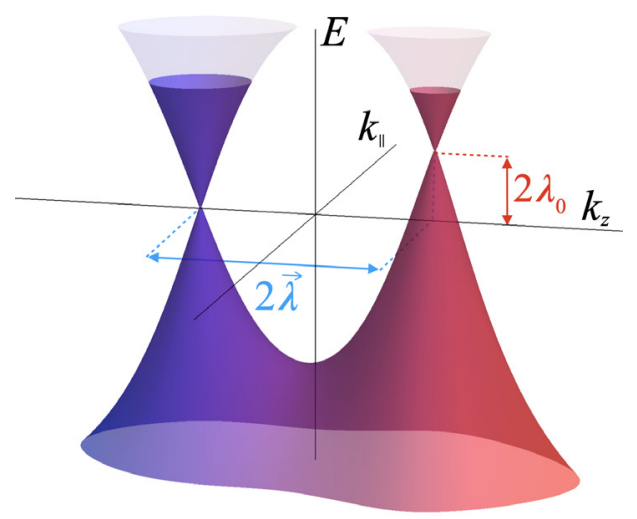

Figure 2. A schematic view of a Weyl semimetal's band structure.

Since the Weyl nodes in the Brillouin zone are separated by the distance $\vec{\lambda}$ and the interaction between the electronic quasiparticles is weak one can effectively treat the Weyl cones independently. The dominant relaxation processes are elastic scattering processes in which momentum of the quasiparticles is randomized and somewhat rarer intervalley scattering processes in which a quasiparticles is scattered from one Weyl cone to another. In this latter case the chirality changes. The relevant relaxation time scale for this process is the intervalley scattering time $\tau_{5}$. This leads to the idea to write down an effective transport theory in which one only keeps track of the particle number and energy of the quasiparticles in each Weyl cone. In a magnetic field and under the application of an electric field and gradients in the chemical potential and temperature one can solve the anomalous transport equations and finds the induced conductivity matrix [21]

$$
\left(\begin{array}{c}
\delta J_{i}^{\epsilon} \\
\delta J_{i}
\end{array}\right)=\Sigma_{i j} \cdot\left(\begin{array}{c}
-\partial_{j} T \\
E_{j}-\partial_{j} \mu
\end{array}\right) .
$$

Here $\left(J_{i}, J_{i}^{\epsilon}\right)$ are the components of the charge current and of the energy current. This result does not depend on the chirality. Both chiralities contribute in the same way. For a gas of free chiral fermions in a weak magnetic field the conductivity matrix is

$$
\Sigma_{i j}=\left(\begin{array}{cc}
\kappa_{i j} & \beta_{i j} \\
\alpha_{i j} & \sigma_{i j}
\end{array}\right),
$$


where the different entries are the anomaly induced contributions to the different conductivities

$$
\begin{aligned}
\text { thermal conductivity : } \kappa_{i j} & =\tau_{5} B_{i} B_{j} \frac{15 \mu^{2} T-5 \pi^{2} T^{3}}{24 \Delta} \\
\text { electro - thermal conductivity : } \beta_{i j} & =\tau_{5} B_{i} B_{j} \frac{11 \pi^{2} T^{2} \mu+15 \mu^{3}}{8 \pi^{2} \Delta} \\
\text { thermo - electric conductivity : } \alpha_{i j} & =-\tau_{5} B_{i} B_{j} \frac{5 \mu T}{4 \Delta} \\
\text { electric conductivity }: \sigma_{i j} & =\tau_{5} B_{i} B_{j} \frac{21 \pi^{2} T^{2}+15 \mu^{2}}{8 \pi^{2} \Delta},
\end{aligned}
$$

with $\Delta=7 \pi^{4} T^{4}+6 \pi^{2} T^{2} \mu^{2}+15 \mu^{4}$. Let us define the heat current

$$
\vec{J}_{Q}=\vec{J}^{\epsilon}-\mu \vec{J}
$$

The thermal conductivity is define throught the heat current by

$$
J_{Q, i}=-\hat{\kappa}_{i j} \partial_{j} T .
$$

It is partcilularly interesting to compute the Lorentz ratio

$$
\frac{\hat{\kappa}_{i i}}{\sigma_{i i}}=\frac{5 k_{B}^{2} \pi^{2}\left(\pi^{2} k_{B}^{2} T^{3}+3 \mu^{2} T\right)}{e^{2} 9\left(7 \pi^{2} k_{B}^{2} T^{2}+5 \mu^{2}\right)}=\frac{\pi^{2} k_{B}^{2}}{3 e^{2}} T+O\left(T^{2} / \mu^{2}\right) .
$$

Here we have restored the unit of electric charge and the Boltzmann constant. In the limit of large chemical potential we see that we recover the Wiedemann-Franz law. This is quite interesting since the anomaly induced currents depend on the anomaly coefficients in (5), (6). For the special case of a single chiral fermion they are just right to reproduce the WiedemannFranz law indicative of the quasiparticle nature. In a similar vein the Mott formula for the Seebeck coefficient can be recovered from anomaly induced transport. Due to the quasiparticle nature these transport formulas can also be calculated from a kinec theory approach [22-24].

For large magnetic field in which the fermions reside completely in the lowest Landau level the conductivity matrix is

$$
\Sigma_{i j}=\frac{\tau_{5}}{4 \pi^{2}|B|}\left(\begin{array}{cc}
\frac{\pi^{2} T}{3} & \mu \\
0 & 1
\end{array}\right) B_{i} B_{j}
$$

Again it can be checked that the Wiedemann-Franz law holds. For intermediate field strengths the conductivities can be calculated by summing over Landau levels in kinetic theory [25].

Generically the formulas predict a strong enhancement of the conductivities at small magnetic field with quadratic increase as the magnetic field is increase. This is indeed seen in experiments such as [26-28].

\section{Torsion}

Spacetime torsion is the geometric property that a parallelogram spanned by parallel vectors does not form a a closed loop [29]. In the Cartan formalism torsion and curvature are expressed via vielbein $e^{a}=e_{\mu}^{z} d x^{\mu}$ and spin connection $\omega_{b}^{a}=\omega_{\mu, b}^{a} d x^{\mu}$ by

$$
\begin{aligned}
d e^{a}+\omega_{b}^{a} e^{b} & =\theta^{a}, \\
d \omega^{a b}+\omega_{c}^{a} \wedge \omega_{b}^{c} & =R^{a b} .
\end{aligned}
$$


It is sometimes stated that there is a torsional contribution to the axial anomaly given by the Nieh-Yan tensor [30]

$$
d\left(e^{a} \wedge \theta_{a}\right)=\theta^{a} \wedge \theta_{a}+R_{a b} \wedge e^{a} \wedge e^{b} .
$$

However, this term has the wrong dimension for an anomaly, it is only of dimension two whereas anomalies have dimension four. This is related to the fact that the vielbein is not a connection but a well defined tensor under local Lorentz transformations and also a well defined one form. Nevertheless it is tempting to conjecture that torsion might induce similar chiral transport phenomena as the chiral magnetic and chiral vortical effects. For a general current $J$ there are two candidate terms

$$
J=c_{T}^{\|} u_{a} u_{b} \theta^{a} \wedge e^{b}+c_{T}^{\perp}\left(\eta_{a b}-u_{a} u_{b}\right) \theta^{a} e^{b}+c_{V} u \wedge d u,
$$

with a priori the right quantum numbers. While a torsional anomaly would be proportional to the square of some UV scale $\Lambda^{2}$ in order to compensate for the wrong dimension, the transport terms might depend on IR scales such as temperature $T$ and chemical potential $\mu$ just as it happens for the CME and CVE currents. We emphasize that at finite temperature and chemical potential one must implicitly specify a frame $u_{a}$ in which the system is at rest. This frame field is essential in writing down all possible torsion dependent response terms in (23). This means that one also has to include in the response terms the chiral vortical effect proportional to $u \wedge d u$ with the frame one-form $u=u_{a} e^{a}$. This is important since in any attempt to compute the transport coefficients $c_{T}^{\|}, c_{T}^{\perp}$ and $c_{V}$ via Kubo formulas there is a certain amount of mixing between them. In a recent calculation [31] this mixing was considered and it was found that for a minimally coupled chiral fermion

$$
c_{T}^{\|}=c_{T}^{\perp}=0 .
$$

This does not mean that torsion has no effect, but it does state that the effect is perfectly accounted for the the chiral vortical effect with transport coefficient $c_{V}$. Indeed in a background with $u_{a}=(1,0,0,0)$, vanishing spin connection but non-trivial vielbein $e^{a}=d x^{a}+\delta e^{a}$ one has to first order in the variation of the vielbein

$$
J=c_{V} d\left(\delta e^{a}\right)=c_{V} \delta \theta^{a} .
$$

One might be tempted to attribute this to a genuine torsional transport, but it is really just a manifestation of the chiral vortical effect due to frame dragging which happens even in spacetimes without torsion [5].

\section{References}

[1] R.A. Bertlmann, Anomalies in quantum field theory (Oxford, UK: Clarendon, 1996)

[2] K. Fujikawa, H. Suzuki, Path integrals and quantum anomalies (2004)

[3] K. Landsteiner, Acta Phys. Polon. 47, 2617 (2016)

[4] J.M. Luttinger, Phys. Rev. 135, A1505 (1964)

[5] K. Landsteiner, E. Megias, F. Pena-Benitez, Anomalies and Transport Coefficients: The Chiral Gravito-Magnetic Effect, in 11th Workshop on Non-Perturbative Quantum Chromodynamics (2011), 1110. 3615

[6] D.T. Son, P. Surowka, Phys. Rev. Lett. 103, 191601 (2009), 0906. 5044

[7] Y. Neiman, Y. Oz, JHEP 03, 023 (2011), 1011.5107

[8] K. Jensen, R. Loganayagam, A. Yarom, JHEP 02, 088 (2013), 1207.5824

[9] M. Stone, J. Kim, Phys. Rev. D98, 025012 (2018), 1804.08668 
[10] S.P. Robinson, F. Wilczek, Phys. Rev. Lett. 95, 011303 (2005), gr-qc/0502074

[11] S. Golkar, S. Sethi, JHEP 05, 105 (2016), 1512 . 02607

[12] N. Yamamoto, Phys. Rev. D92, 085011 (2015), 1502.01547

[13] A. Gynther, K. Landsteiner, F. Pena-Benitez, A. Rebhan, JHEP 02, 110 (2011), 1005.2587

[14] D.E. Kharzeev, L.D. McLerran, H.J. Warringa, Nucl. Phys. A 803, 227 (2008), Q711.0950

[15] K. Fukushima, D.E. Kharzeev, H.J. Warringa, Phys. Rev. D 78, 074033 (2008), 0808.3382

[16] D.E. Kharzeev, J. Liao, Nature Rev. Phys. 3, 55 (2021), 2102.06623

[17] J.K. Ghosh, S. Grieninger, K. Landsteiner, S. Morales-Tejera, Phys. Rev. D 104, 046009 (2021), 2105.05855

[18] M. Abdallah et al. (STAR) (2021), 2109.00131

[19] N.P. Armitage, E.J. Mele, A. Vishwanath, Rev. Mod. Phys. 90, 015001 (2018)

[20] H.B. Nielsen, M. Ninomiya, Phys. Lett. B 130, 389 (1983)

[21] M.N. Chernodub, Y. Ferreiros, A.G. Grushin, K. Landsteiner, M.A.H. Vozmediano (2021), 2110.05471

[22] D.T. Son, B.Z. Spivak, Phys. Rev. B 88, 104412 (2013), 1206. 1627

[23] B.Z. Spivak, A.V. Andreev, Phys. Rev. B 93, 085107 (2016)

[24] R. Lundgren, P. Laurell, G.A. Fiete, Phys. Rev. B 90, 165115 (2014)

[25] K. Das, A. Agarwal, Phys. Rev. Research 2, 013088 (2020)

[26] Q. Li, D.E. Kharzeev, C. Zhang, Y. Huang, I. Pletikosic, A.V. Fedorov, R.D. Zhong, J.A. Schneeloch, G.D. Gu, T. Valla, Nature Phys. 12, 550 (2016), 1412. 6543

[27] J. Gooth, A.C. Niemann, T. Meng, A.G. Grushin, K. Landsteiner, B. Gotsmann, F. Menges, M. Schmidt, C. Shekhar, V. Süß et al., Nature 547 (2017)

[28] D. Vu, W. Zhang, C. Şahin, M.E. Flatté, N. Trivedi, J.P. Heremans, Nature Materials (2021)

[29] F.W. Hehl, P. von der Heyde, G.D. Kerlick, J.M. Nester, Rev. Mod. Phys. 48, 393 (1976)

[30] H. Nieh, C. Yang, Int. J. Mod. Phys. A 22, 5237 (2007)

[31] Y. Ferreiros, K. Landsteiner, arXiv e-prints arXiv:2011.10535 (2020), 2011.10535 